\title{
COMPARATIVE ANALYSIS OF RCM MECHANISMS BASED ON PARALLELOGRAM USED IN SURGICAL ROBOTS FOR LAPAROSCOPIC MINIMALLY INVASIVE SURGERY
}

\author{
Roman Trochimczuk \\ Bialystok University of Technology, Department of Automatic Control and Robotics, Bialystok, Poland \\ e-mail: r.trochimczuk@pb.edu.pl
}

\begin{abstract}
This article presents results of comparative analysis of kinematics and dynamics of five varieties of the parallelogram-based RCM mechanism applied in real-life designs of surgical robots. Analyses were conducted using ANSYS Workbench v. 16.2. Obtained results allow for formulation of guidelines concerning conscious selection of the form of the RCM mechanism and assessment of its usefulness from the perspective of application in new solutions of laparoscopic surgical robots.
\end{abstract}

Keywords: RCM mechanism, parallelogram, surgical robots, MIS, FEM simulation

\section{Introduction}

Since the beginning of the 90's, minimally invasive operations have become very popular and widely performed surgical procedures as an alternative to traditional opening of the abdominal wall. The main advantages of such procedures are, above all: smaller post-operative wounds and lesser pain linked to their treatment, faster convalescence after the procedure, and the possibility of a quicker return to health and everyday professional activity, a lower number of infections in comparison to classical methods, lower cost of treatment related to shorter hospitalization, and faster return from medical leave as well as a better cosmetic effect. These techniques are successfully applied today in, among other fields, neurosurgery, urology, vascular surgery, cardiac surgery, laryngology and orthopedics as well as many other disciplines of interventional medicine (Kuo and Dai, 2009; Kuo et al., 2012). In the classical procedure, laparoscopy requires the doctor to have many hours of pre-operative training, proper posture and hand positioning for the purpose of operating the instrument. The great difficulty in each of the techniques listed is, above all: non-intuitive performance of precise mirror movements with the instrument, narrow field of vision of the endoscope as well as the method of removing excised organs. The size and shape of actual tools used during operations and a schematic presentation of the idea of a typical laparoscopic procedure are presented in Fig. 1. The development of new technologies in the field of mechatronics and biomedical engineering has resulted in the fact that surgical robots assisting surgeons in the operating room - e.g. the da Vinci robot (Taylor et al., 2016) - currently achieve the greatest accuracy in performing the minimally invasive procedure.

Due to the specific nature of laparoscopic surgery, there is a need to work out a mechanism for robots of this type that through a fixed geometric Remote Center of Motion (RCM) found outside of the mechanism itself, will allow for problem-free operation of the surgical instrument, inserted under the skin through small incisions (Fu et al., 2013; Pan et al., 2014; Roh et al., 2015; Chen et al., 2015; Chng et al., 2016; Li et al., 2017; Lee et al., 2018; Bai et al., 2014; Yip et al., 2014). Many scientific centers around the world are currently working on the parallelogram mechanisms of this type, as can be seen in studies (Zong et al., 2008; Koseki et al., 2003; Choi 
(a)

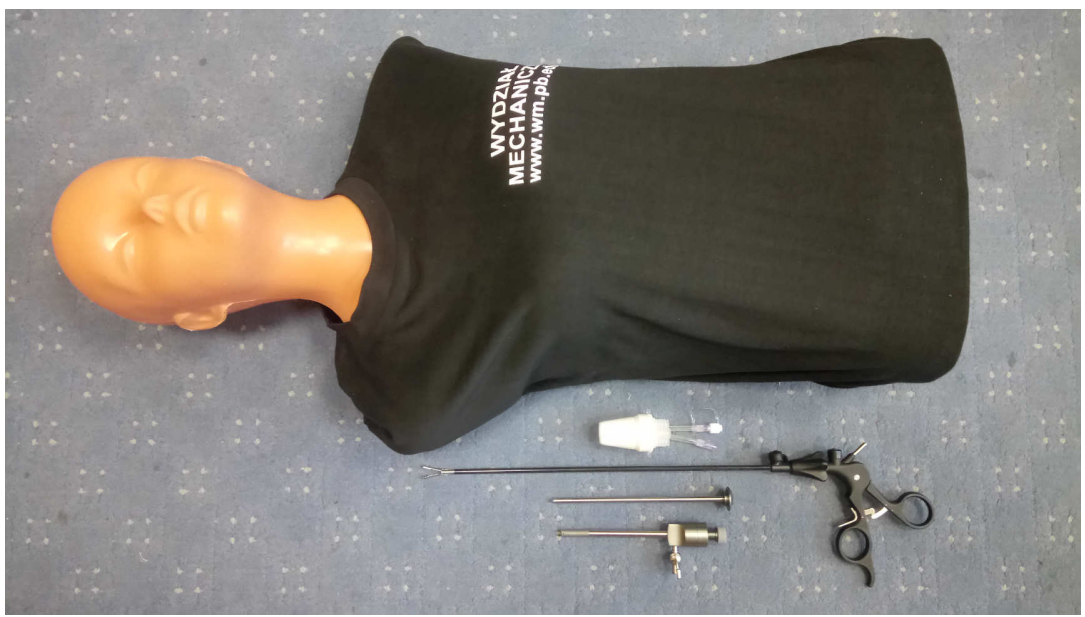

(b)

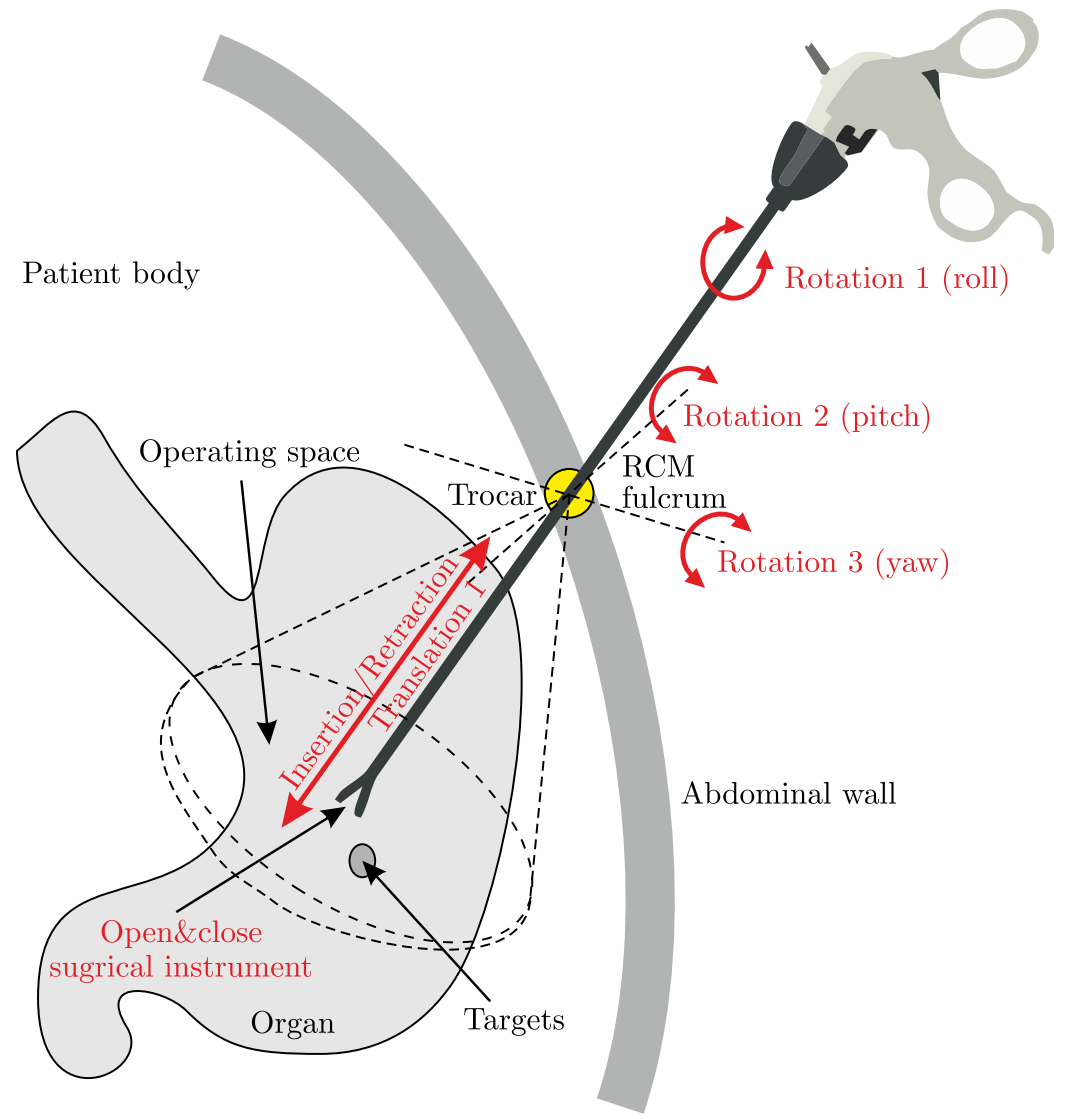

Fig. 1. View of (a) typical size of surgical instruments use in laparoscopic surgery, (b) idea of laparoscopy surgery

et al., 2013; Zhang et al., 2015; Hadavand et al., 2014). However, analyses determining the suitability of applying solutions of this type in original designs are absent in these studies.

This paper presents the results of comparative analysis of five varieties of the parallelogram-based RCM mechanism most commonly applied in real-life designs of surgical robots. Analyses are conducted using ANSYS Workbench v. 16.2. The results of analysis are intended to contribute to better determination of usefulness of a given type of the RCM mechanism from the perspective of its adoption in new surgical robot arm designs. This work constitutes a continuation, expansion and supplementation of the knowledge described in the author's work (Trochimczuk, 2013; Trochimczuk et al., 2019). 


\section{Materials and methods}

In modern surgical robot solutions, a remote center of motion is provided by three methods: i) through passive point ( $\mathrm{RCM}$ ) fixation, ii) kinematic point fixation (hardware method), and iii) active point fixation (mechatronic control method) (Trochimczuk et al., 2019). The first of these methods is the least popular and essentially concerns only the simplest robot designs. The second is the most commonly encountered due to use of multiple varieties of the spherical mechanism and the parallelogram mechanism. The third method is used, as a rule, with the application of existing structures of industrial robots adapted to medical tasks.

The research problem undertaken in this article was formulated through definition, based on reports in the literature, of five most commonly applied varieties of the parallelogram-based RCM mechanism used to position the laparoscopic surgical instrument. Thanks to its simple structure and high rigidity and, hence, high accuracy of the end effector movements, it is a very popular design that has been successfully adopted by many designers in new surgical robot solutions. Examples of applications are discussed in the works (Trochimczuk, 2013; Wang et al., 2016). While reviewing the literature from the perspective of the usefulness of applied solutions, the author noticed that there is a lack of descriptions confirming justification of an applied kinematic solution and providing a guide for designing new constructions. A list of analyzed results of studies is given in (Gijbels et al., 2014; Liu and Wang, 2003). The five configurations of the kinematic structure selected for the purposes of original research are presented in Fig. 2.

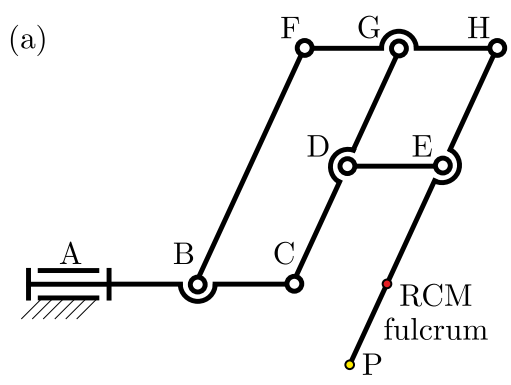

(d)

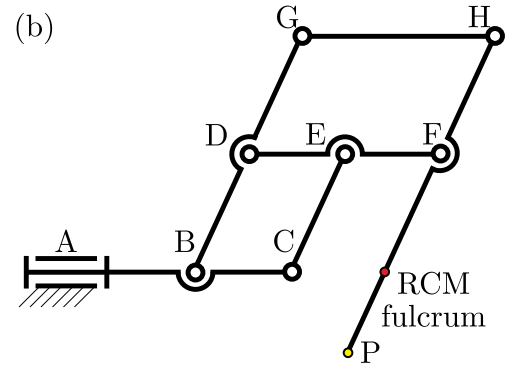

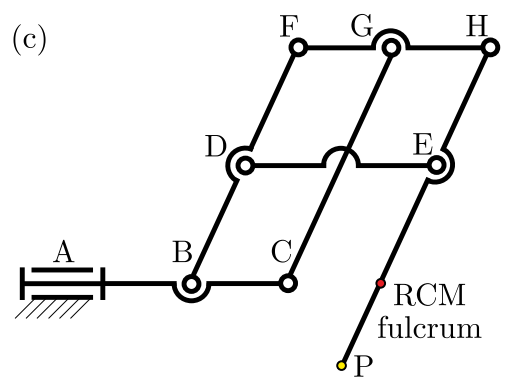

(c)

(e)

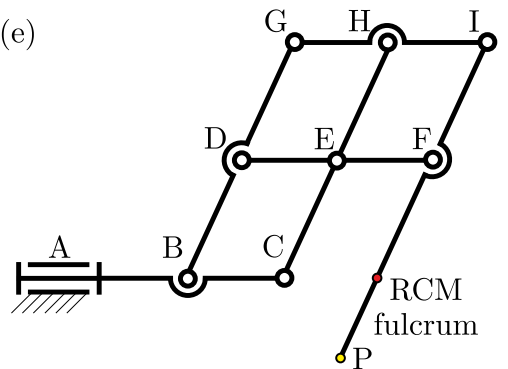

Fig. 2. Different structure of parallelogram RCM mechanisms: (a) configuration A, (b) configuration B, (c) configuration $\mathrm{C},(\mathrm{d})$ configuration $\mathrm{D}$, (e) configuration E

For the purposes of analysis, it is accepted that the drives for moving the parallelogram are situated, in each of the configurations, in kinematic pairs designated as A and B in Fig. 2. A three-dimensional solid model was made for each kinematic structure in SolidWorks 2018 software. These models were then imported into ANSYS Workbench v. 16.2 software (Fig. 3).

Stainless steel taken from the standard ANSYS library (Ansys, 2013) was accepted as the material of all links of the mechanisms. A list of the weights of individual links made of this material is given in Table 1 . The following designations are adopted in the table: link 1 - weight of the fixation link; link 2 - weight of the link forming the rotary pair A with the fixation link; 


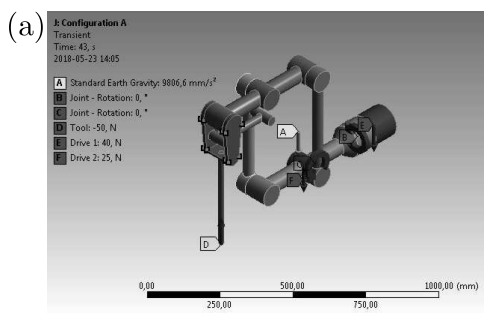

(d)
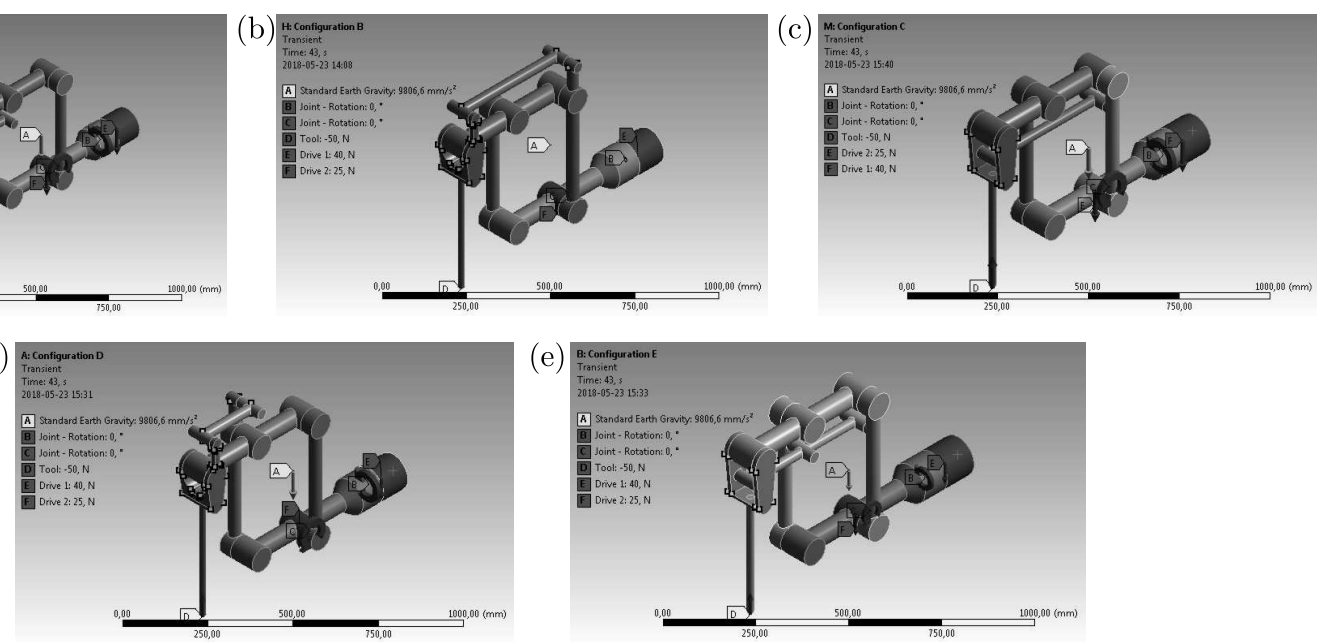

Fig. 3. View of solid models in ANSYS Workbench: (a) configuration A, (b) configuration B, (c) configuration $\mathrm{C},(\mathrm{d})$ configuration $\mathrm{D}$, (e) configuration E

link 3 - weight of the moving link forming the rotary pair B with link 2 ; link 4 - weight of the 2 nd moving link forming a rotary pair with link 2 parallel to link 3 ; link 5 - weight of the link parallel to link 2 ; link 6 - weight of the link forming a rotary pair with link 5 , serving for fixation of the surgical instrument; link 7 - weight of the link (surgical instrument); link 8 - weights of additional links differentiating individual structures of the mechanism. For the purposes of numerical simulations, constant loads were also assumed at the points: $\mathrm{A}=40 \mathrm{~N}$, $\mathrm{B}=25 \mathrm{~N}$, acting according to the direction of gravity, arising from the weight of actual drives used to move pairs of the mechanism (solutions from Harmonic Drive - FHA-17C and FHA-25C - rotary actuators were adopted) as well as variable load at the TCP of the surgical instrument $P= \pm 50 \mathrm{~N}$ simulating the maximum loading during resection or cutting of an organ. The action of the force of gravity on the constructions was also simulated. The following parameters were accepted in every rotary joint of kinematic pairs: torsional stiffness $=0.005 \mathrm{~N} \cdot \mathrm{mm} / \mathrm{deg}$ and torsional damping $=0.01 \mathrm{~N} \cdot \mathrm{mm} \cdot \mathrm{s} / \mathrm{deg}$. Additionally, it was assumed that movement in the rotary pairs (points A and B) was restricted to the range of $\pm 45^{\circ}$. Models prepared in this way were subjected to further kinematic and dynamic analysis. For this purpose, modules of ANSYS Workbench software were employed: 1) rigid dynamics, 2) modal and 3) harmonic response (Ansys, 2013).

Table 1. Mass of links in selected mechanism configurations

\begin{tabular}{|l|c|c|c|c|c|}
\hline & Config. A & Config. B & Config. C & Config. D & Config. E \\
\hline \hline Total mass $[\mathrm{kg}]$ & 52.1 & 55.578 & 52.722 & 54.624 & 53.122 \\
\hline Link 1 [kg] & 7.5477 & 7.5477 & 7.5477 & 7.5477 & 7.5477 \\
\hline Link 2 [kg] & 18.075 & 18.075 & 18.075 & 18.075 & 18.075 \\
\hline Link 3 [kg] & 4.687 & 5.195 & 4.76 & 4.687 & 4.76 \\
\hline Link $4[\mathrm{~kg}]$ & 4.76 & 4.687 & 4.687 & 5.195 & 4.76 \\
\hline Link 5 [kg] & 11.415 & 11.415 & 11.415 & 11.415 & 11.415 \\
\hline Link 6 [kg] & 3.4287 & 4.5317 & 3.4287 & 5.4317 & 3.4287 \\
\hline Link $7[\mathrm{~kg}]$ & 0.92315 & 1.0842 & 0.92315 & 1.0842 & 0.92315 \\
\hline Link 8 [kg] & 1.2639 & 3.043 & 1.8856 & 2.0885 & 2.2123 \\
\hline
\end{tabular}




\subsection{Kinematic analysis of parallelogram mechanisms}

For the purposes of assessing the kinematics of the parallelogram mechanism, tests of changes of the TCP position of the surgical instrument end effector as well as tests of changes of TCP velocity and acceleration were performed in the ANSYS Rigid Dynamics module on the assumption of displacements of links in the mechanism according to the adopted scenario: rotation by angle $+45^{\circ}$ over time of $1 \mathrm{~s}$ in the rotary pair designated as $\mathrm{A}$; return to initial position over time of $1 \mathrm{~s}$; rotation by angle $-45^{\circ}$ over time of $1 \mathrm{~s}$ in the rotary pair designated as $\mathrm{A}$ in Fig. 2 ; return to the initial position over time of $1 \mathrm{~s}$. Next, the same scenario is repeated with respect to the rotary pair designated as B in Fig. 2. After such displacement, simultaneous motion occurs in the rotary pairs A and B according to the scenarios described above. The phase of the full scheme of movements lasts 48 seconds, and over its duration, a force of $+50 \mathrm{~N}$ with an orientation opposite to the force of gravity acts on the end effector for a time of $24 \mathrm{~s}$, and for the next 24 seconds, a force of $-50 \mathrm{~N}$ oriented consistently with the force of gravity. This test was conducted once for configuration A, since each of the designed mechanisms would have the same motion due to identical geometric sizes of the links making up the parallelogram.

To describe the forward kinematics task of the parallelogram mechanism the $\mathrm{D}-\mathrm{H}$ notation is used. A manipulator in its basic configuration (without additional links differing in the analyzed configurations) is analyzed. The procedure described below is analogous with respect to all manipulator configurations defined in the paper. We obtain a transformation matrix for the parallelogram mechanism to configuration A (Fig. 2a)

$$
{ }_{P}^{A} \mathbf{T}=\left[\begin{array}{cccc}
A_{11} & A_{21} & -s \theta_{1} & A_{41} \\
A_{12} & A_{22} & 0 & A_{42} \\
A_{13} & A_{23} & 0 & A_{43} \\
0 & 0 & 0 & 1
\end{array}\right]
$$

where ${ }_{P}^{A} \mathbf{T}$ is transformation matrix of the $\mathrm{P}$ point coordinate system relative to A point coordinate system, and

$$
\begin{aligned}
& A_{11}=c\left(\beta+\theta_{2}\right) c \theta_{1} c \theta_{3} c \theta_{4}-c\left(\beta+\theta_{2}\right) c \theta_{1} s \theta_{3} s \theta_{4} \\
& A_{12}=c\left(\beta+\theta_{2}\right) c \theta_{3} c \theta_{4} s \theta_{1}-c\left(\beta+\theta_{2}\right) s \theta_{1} s \theta_{3} s \theta_{4} \\
& A_{13}=s\left(\beta+\theta_{2}\right) s \theta_{3} s \theta_{4}-c\left(\beta+\theta_{2}\right) c \theta_{3} c \theta_{4} \\
& A_{21}=-c\left(\beta+\theta_{2}\right) c \theta_{1} c \theta_{3} s \theta_{4}-c\left(\beta+\theta_{2}\right) c \theta_{1} c \theta_{4} s \theta_{3} \\
& A_{22}=-c\left(\beta+\theta_{2}\right) c \theta_{3} s \theta_{1} s \theta_{4}-c\left(\beta+\theta_{2}\right) c \theta_{4} s \theta_{1} s \theta_{3} \\
& A_{23}=s\left(\beta+\theta_{2}\right) c \theta_{3} s \theta_{4}+s\left(\beta+\theta_{2}\right) c \theta_{4} s \theta_{3} \\
& A_{41}=\left[c\left(\beta+\theta_{2}\right) c \theta_{1} c \theta_{3} c \theta_{4}-c\left(\beta+\theta_{2}\right) c \theta_{1} s \theta_{3} s \theta_{4}\right] l_{4}+c\left(\beta+\theta_{2}\right) c \theta_{1} l_{2}+c\left(\beta+\theta_{2}\right) c \theta_{1} c \theta_{3} l_{3} \\
& A_{42}=\left[c\left(\beta+\theta_{2}\right) c \theta_{3} c \theta_{4}-s\left(\beta+\theta_{2}\right) s \theta_{3} s \theta_{4}\right] l_{4}-s\left(\beta+\theta_{2}\right) c \theta_{3} l_{3}-s\left(\beta+\theta_{2}\right) l_{2} \\
& A_{43}=l_{1}-\left[s\left(\beta+\theta_{2}\right) c \theta_{3} c \theta_{4}-s\left(\beta+\theta_{2}\right) s \theta_{3} s \theta_{4}\right] l_{4}-s\left(\beta+\theta_{2}\right) c \theta_{3} l_{2}-s\left(\beta+\theta_{2}\right) l_{2}
\end{aligned}
$$

where

$$
c \theta_{i}=\cos \theta_{i} \quad s \theta_{i}=\sin \theta_{i}
$$

and $\theta_{i}$ is the angle from $X_{i-1}$ to $X_{i}$ measured about $Z_{i}, \theta_{1}$ - angle of rotation in A, $\theta_{2}$ - angle of rotation in $\mathrm{C}, \theta_{3}=90^{\circ}-\left(\theta_{2}+\beta\right), \theta_{4}=-\beta, \beta$ - angle of inclination of the H-P link over $90^{\circ}$ with respect to A-C link, $l_{1}$ - distance from A to $\mathrm{C}$ measured along $Z$ axis, $l_{2}$ - distance from $\mathrm{C}$ to $\mathrm{G}$ measured along $X$ axis, $l_{3}$ - distance from $\mathrm{G}$ to $\mathrm{H}$ measured along $X$ axis, $l_{4}$-distance from $\mathrm{H}$ to $\mathrm{P}$ measured along $X$ axis. 


\subsection{Dynamic analysis of parallelogram mechanisms}

For the purpose of comparing the dynamics of five forms of the parallelogram mechanism, a test was performed for assessing changes in the values of potential energy, kinetic energy, total energy and internal energy over the duration of the mechanisms motion, according to the motion scheme described in Section 2.1. The Ansys Rigid Dynamic module was used for this purpose. Kinetic energy due to motion of parts in a transient analysis is calculated as $0.5^{*}$ omega $T^{*}$ Inertia*omega for rotations. Potential energy is calculated as the sum of the potential energy due to gravity and the elastic energy stored in springs. The potential energy due to gravity is proportional to the height of the body with respect to a reference ground. External energy is calculated as the sum of all the energy the loads and joints bring to a system. The total energy is the sum of potential, kinetic and external energies in the Rigid Dynamics analysis (Ansys, 2013). Other parameters of the test were the same as in the test described in Section 2.1.

\subsection{Modal (natural frequency) analysis of parallelogram mechanisms}

For the purpose of comparing different structures of the parallelogram mechanism, a comparison of the frequency of natural vibrations was also conducted for each of the constructions, forming the basis for further investigations of dynamics. The Ansys Modal module was used, in which linear analysis was performed. It was assumed that only such test results corresponding to the practical ranges of use of the assumed drives moving a given construction would be selected, i.e. within the range from $1 \mathrm{~Hz}$ to $100 \mathrm{~Hz}$, or 1-100 rotation/s. For the purposes of simulation, a finite element mesh was generated automatically in the case of configuration A, yielding: 16497 nodes and 6695 elements; in the case of configuration B: 18510 nodes and 7779 elements; in the case of configuration C: 17097 nodes and 7016 elements; in the case of configuration D: 18097 nodes and 7582 elements; in the case of configuration E: 17804 nodes and 7183 elements. A fixed support is defined on the wall of link 1.

\subsection{Amplitude and phase response analysis of parallelogram mechanisms}

In order to assess the amplitude response and phase response with respect to frequency, as well as to evaluate the values of the resultant deformations, the results of the previous test in the Ansys Modal module were used by linking the given module to the Ansys Harmonic Response module. In this analysis, all loads as well as the structure response vary sinusoidally at the same frequency. A typical harmonic analysis calculates the response of the structure to cyclic loads over a frequency range (a sine sweep) and obtains a graph of some response quantity displacements versus frequency (Ansys, 2013). Harmonic analysis are used to verify whether or not the design structure will successfully overcome resonance, fatigue and other harmful effects of forced vibration (Ansys, 2013). Loads in the kinematic pairs A and B resulting from the action of drive weights and the load of the surgical instrument were simulated in the same manner as described in Sections 2.1 and 2.2. Responses were obtained for frequencies from 1 to $100 \mathrm{~Hz}$, with a step of $1 \mathrm{~Hz}$. A fixed support is defined on the wall of link 1.

\section{Results}

As a result of conducted simulations of operation with the use of ANSYS Workbench v. 16.2 software, sets of results were obtained. On their basis, charts comparing the constructions of parallelogram RCM mechanisms were made. These results were presented in the form of charts using Microsoft Excel 2010 and Matlab 2015a software. 
Figures 4-6 present the results of assessment of the change in position, velocity and acceleration of TCP on the $X, Y$ and $Z$ axes of the surgical instrument, in the chart form, for each structure with respect to the specific instant of time in which such motion is performed.

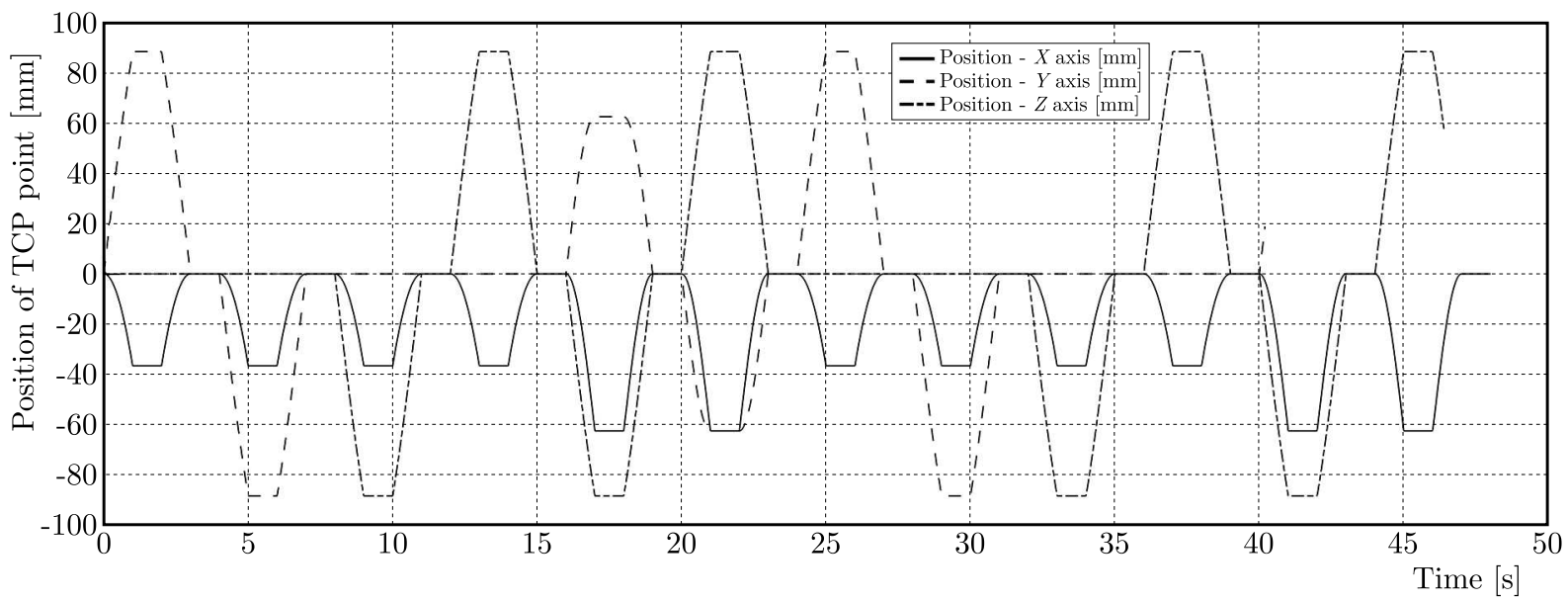

Fig. 4. Position of TCP point of surgical instrument in $X, Y, Z$ axis

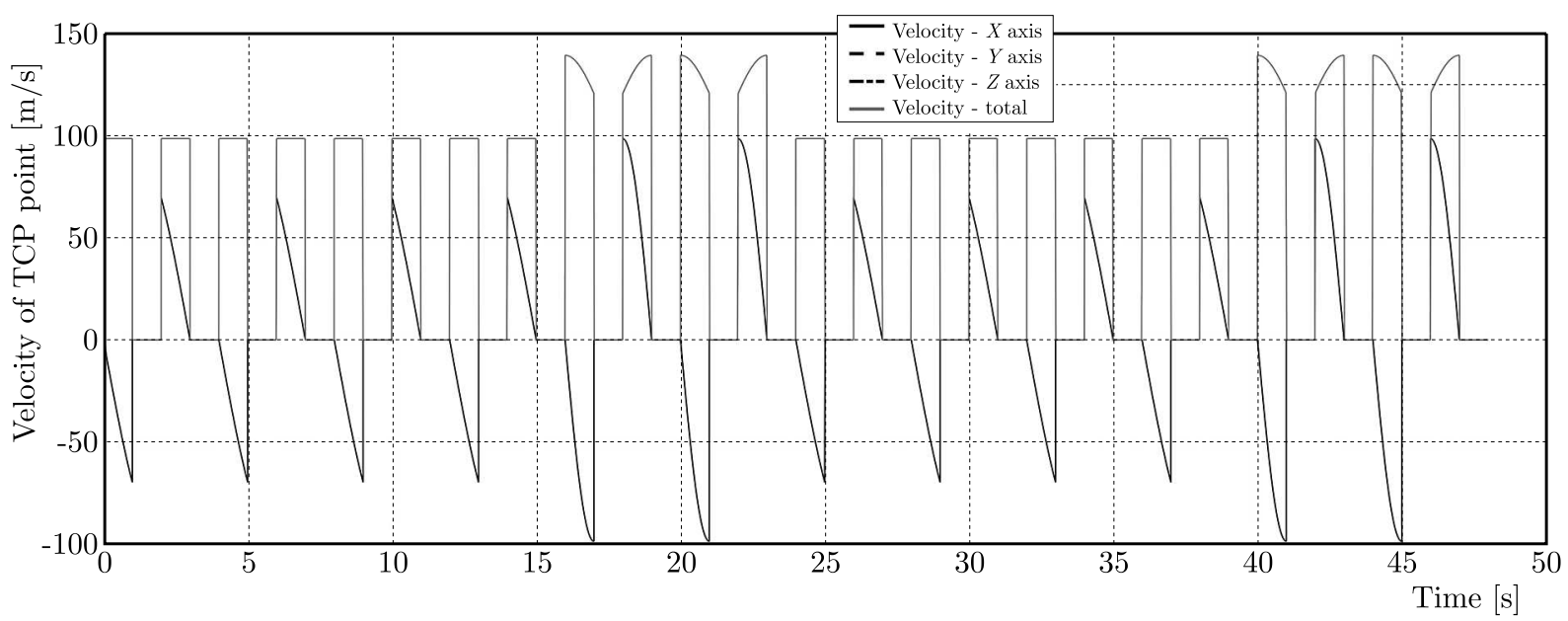

Fig. 5. Velocity of TCP point of surgical instrument in $X, Y, Z$ axis and total velocity

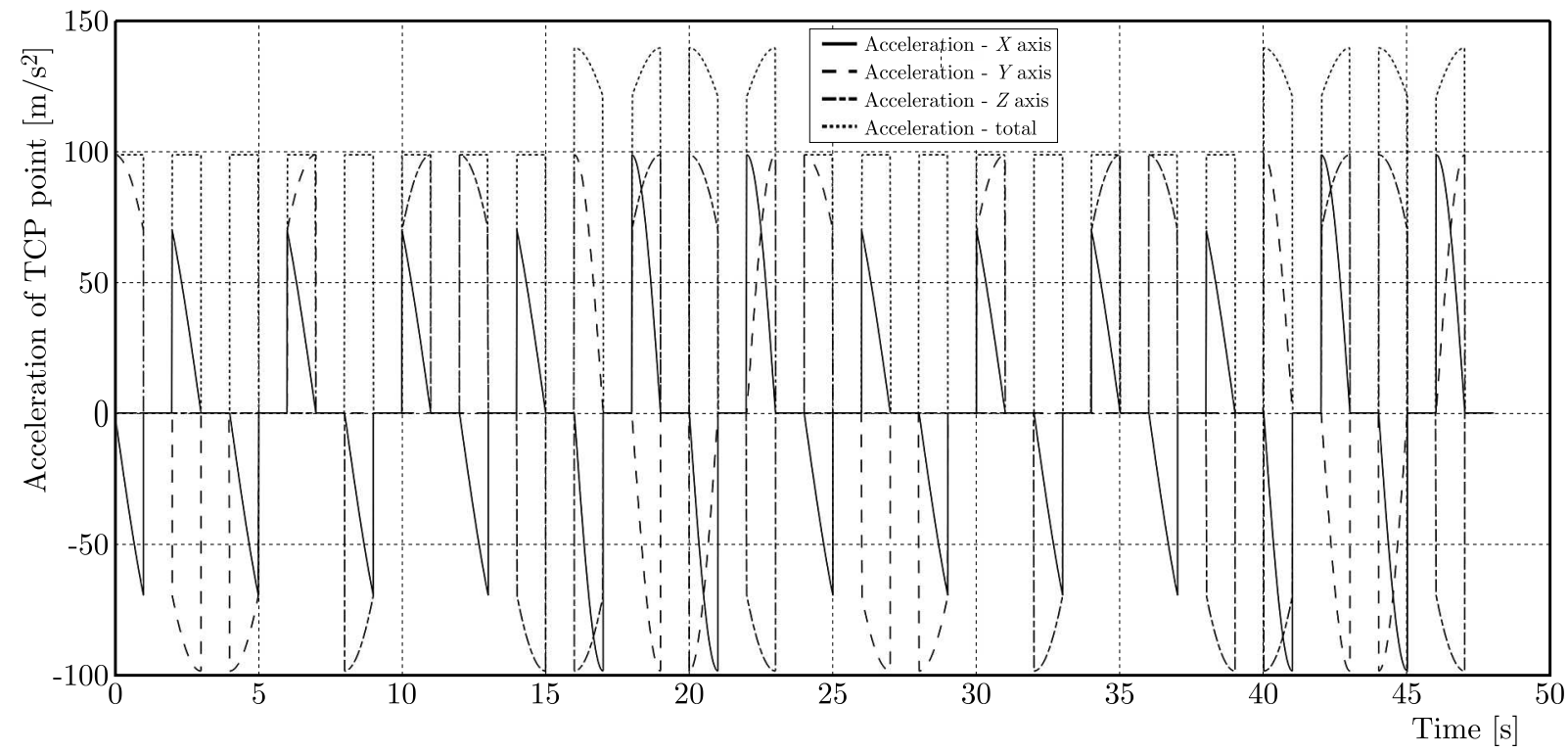

Fig. 6. Acceleration of TCP point of surgical instrument in $X, Y, Z$ axis and total acceleration 
Figures 7-10 contain charts presenting a comparison of changes in potential, kinetic, total and external energy in the tested configurations A-E.

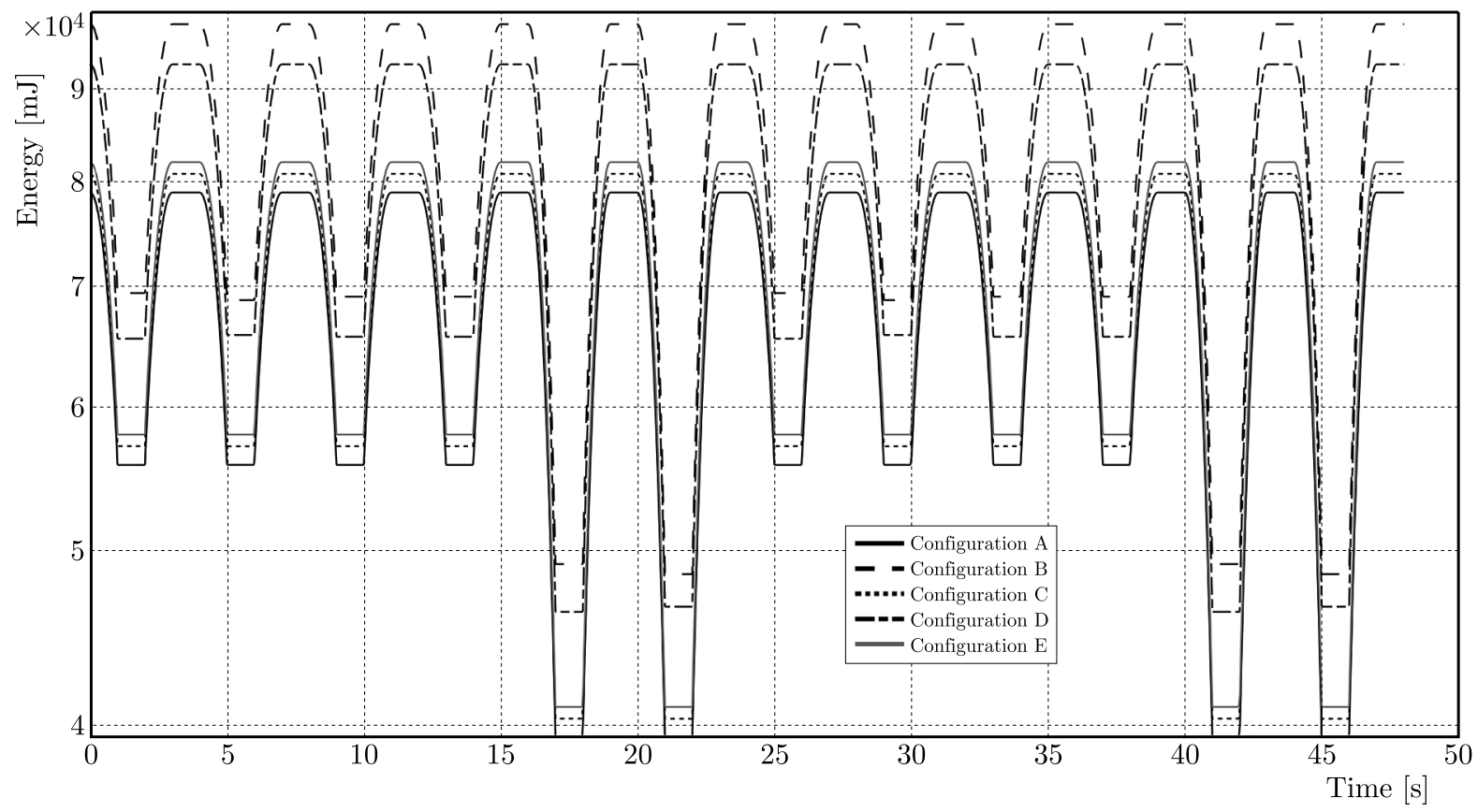

Fig. 7. Graphs of results of comparative analysis of potential energy of all RCM mechanisms

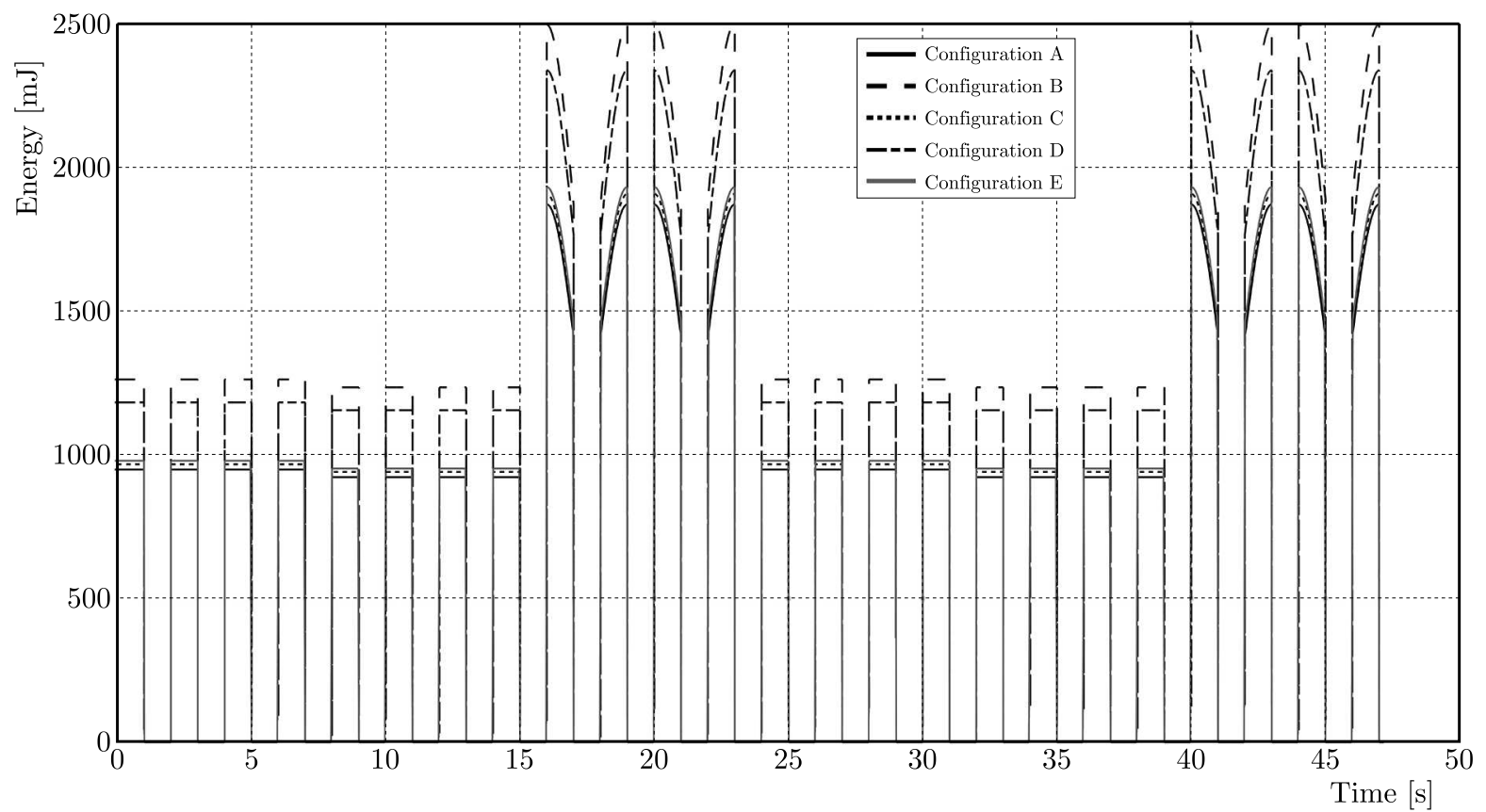

Fig. 8. Graphs of results of comparative analysis of kinetic energy of all RCM mechanisms 


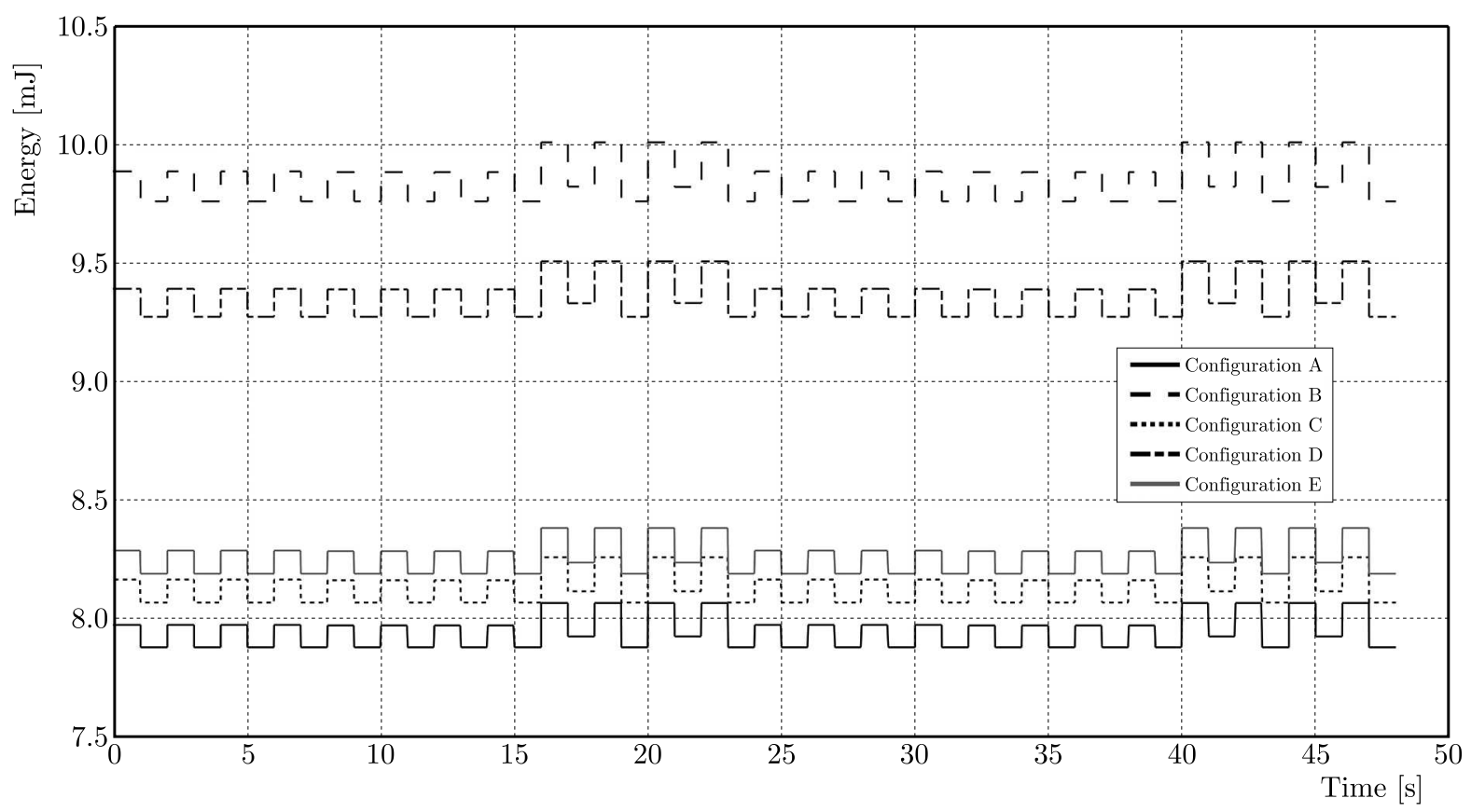

Fig. 9. Graphs of results of comparative analysis of total energy of all RCM mechanisms

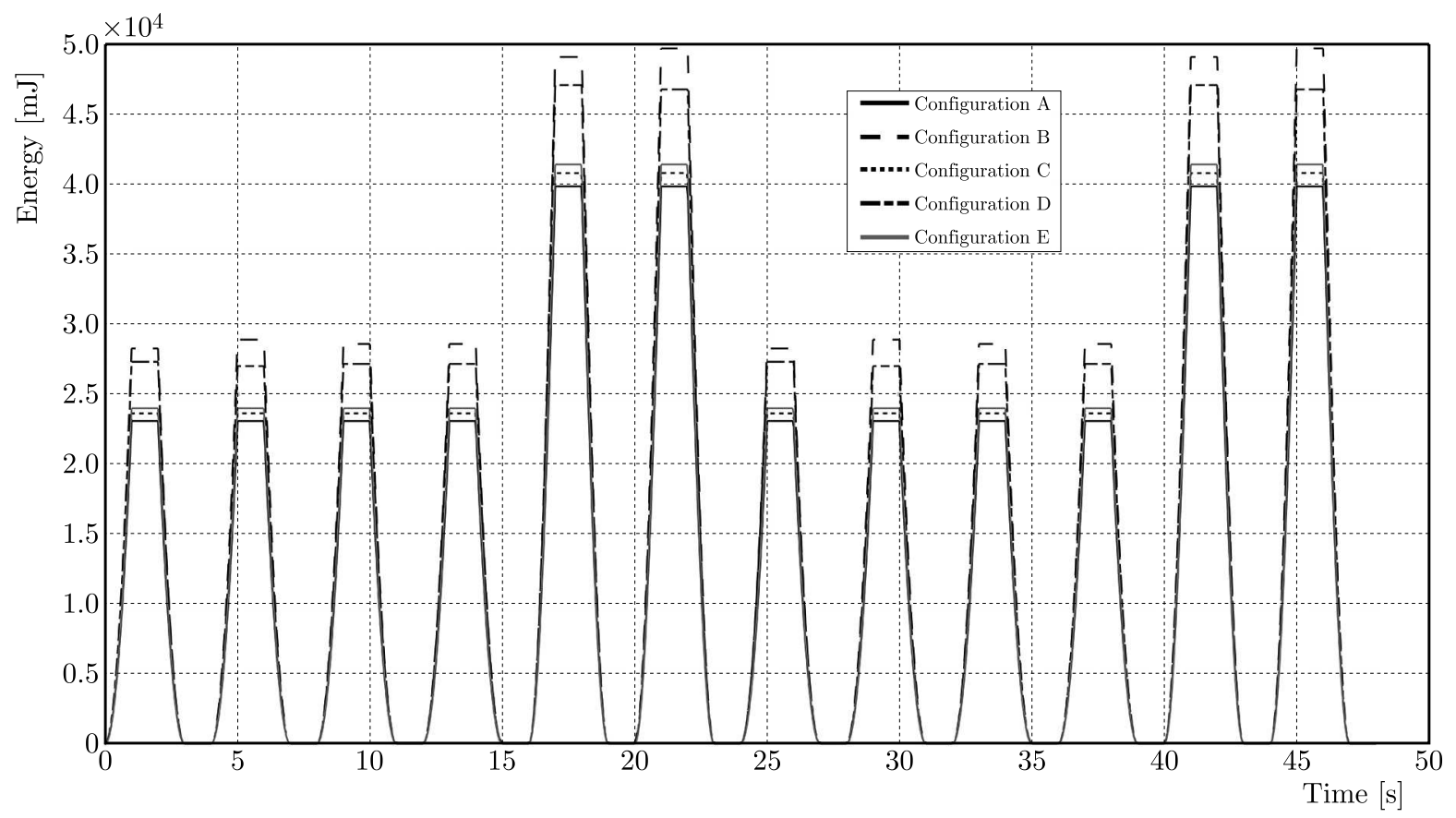

Fig. 10. Graphs of results of comparative analysis of external energy of RCM mechanisms

Figure 11 compares maximum values of potential, kinetic, total and internal energy in each of the tested configurations.

Figures 12 and 13 present the results of the frequency response under loads acting on the end effector with values of $+50 \mathrm{~N}$ and $-50 \mathrm{~N}$, respectively.

Figure 14 presents the results of conducted modal analysis of the parallelogram mechanism in the tested configurations AE.

Figure 15 presents the response of the construction in the form of deformations to a periodic sine input function. 


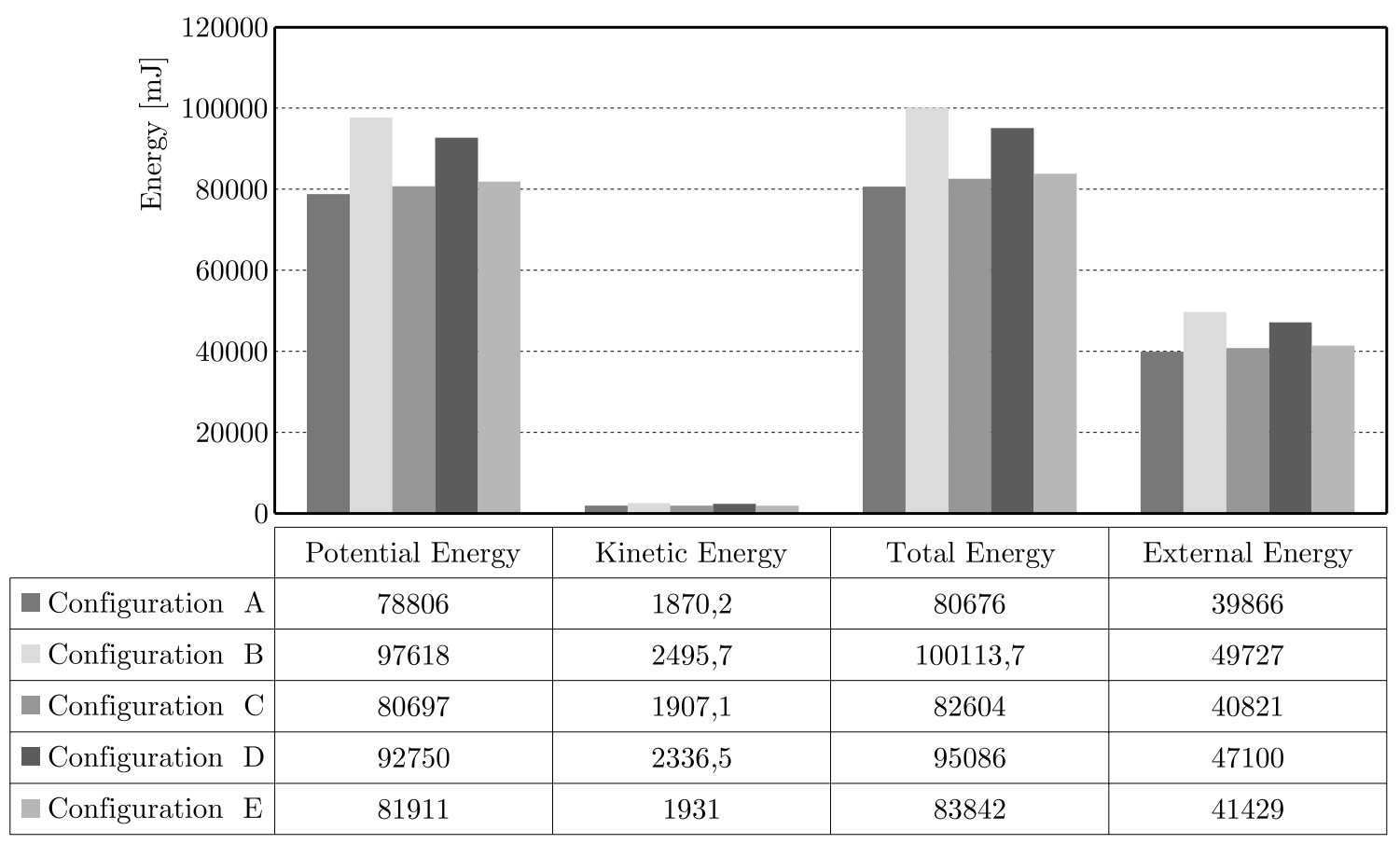

Fig. 11. Graphs of comparative analysis of the maximum value of energy of all RCM mechanisms
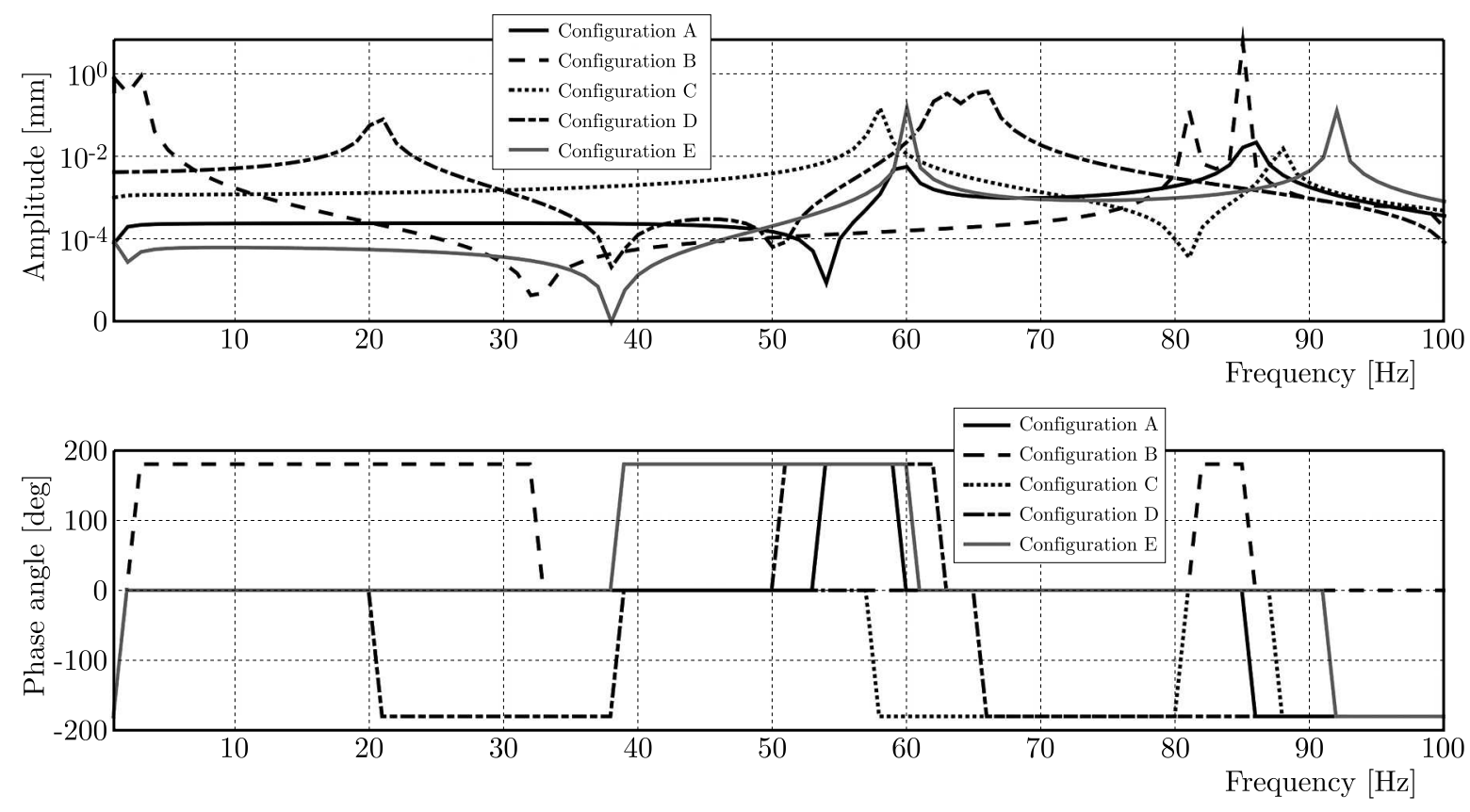

Fig. 12. Graphs of the comparative frequency response of all RCM mechanisms - force $+50 \mathrm{~N}$

\section{Discussion}

- Tests of the kinematics of a multi-link mechanism in ANSYS software (e.g. determination of the position, velocity and acceleration of a selected point of the structure) make it possible to rationally plan a strategy for future control of the mechanism. In the case of the paralellogram RCM mechanism, regardless of the adopted configuration, we obtain the same result of displacement, velocity and acceleration of TCP of the surgical instrument. It is rational to supplement kinematic testing of the RCM mechanism with static numerical tests of stresses, rigidity and deformations of the structure under the action of 

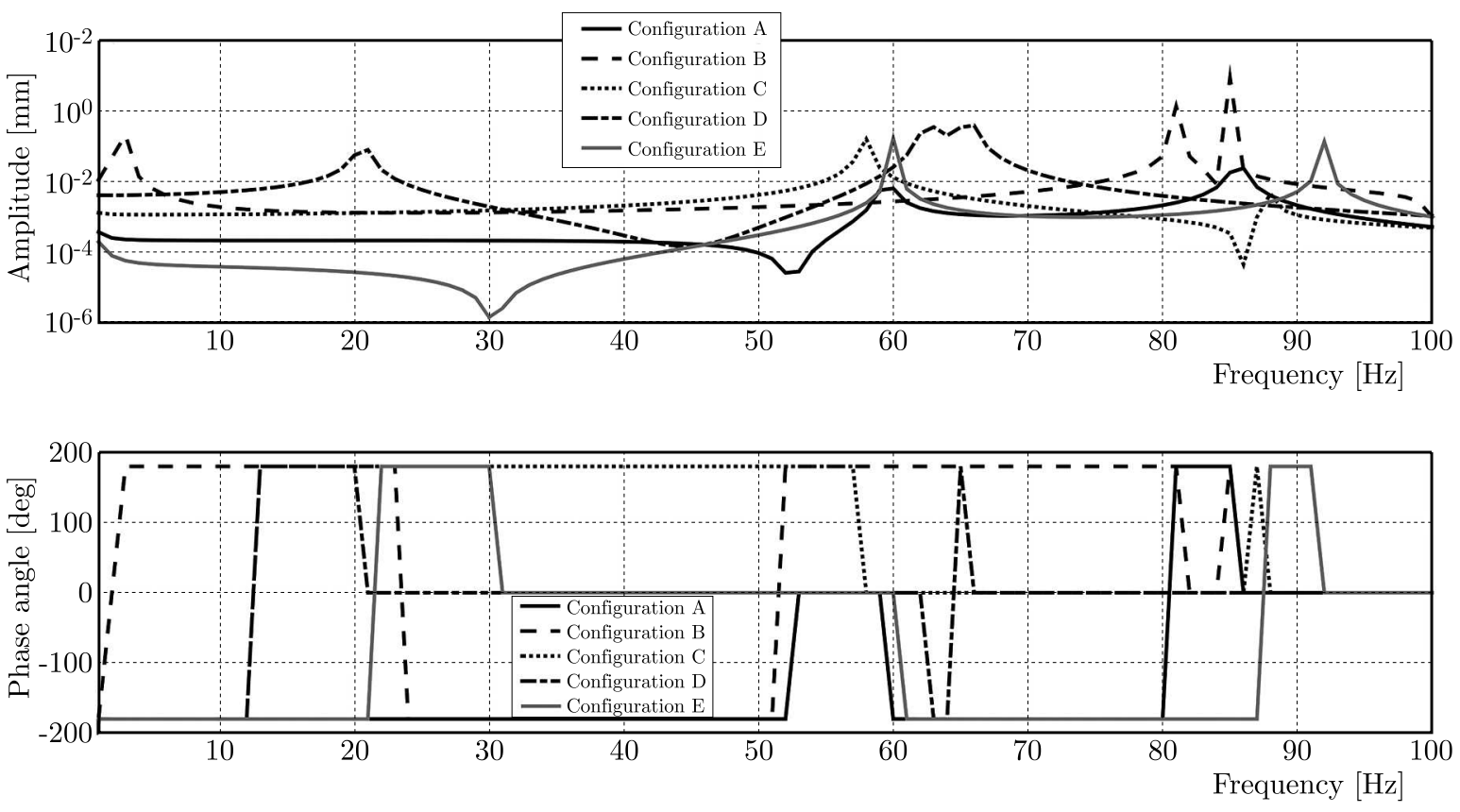

Fig. 13. Graphs of the comparative frequency response of all RCM mechanisms - force $-50 \mathrm{~N}$

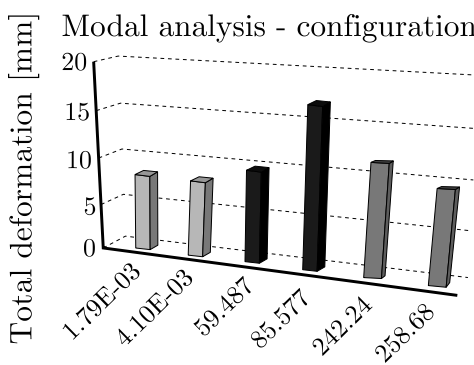

Frequency $[\mathrm{Hz}]$

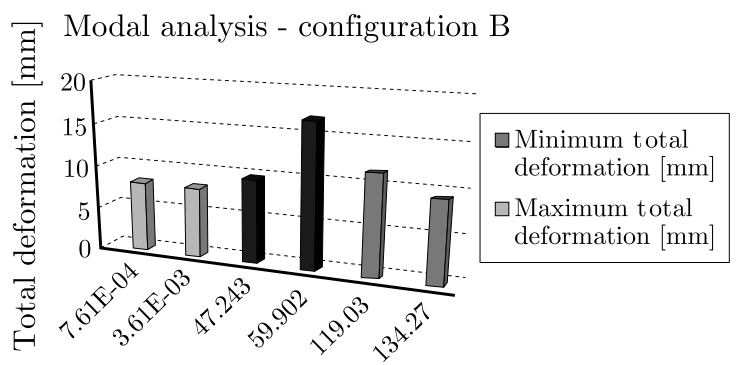

Frequency $[\mathrm{Hz}]$

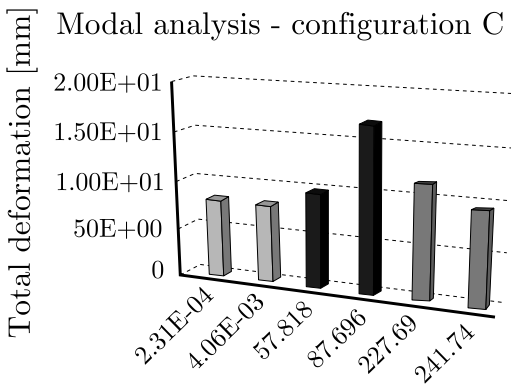

Frequency $[\mathrm{Hz}]$

$$
\begin{aligned}
& \square \text { Minimum total } \\
& \text { deformation }[\mathrm{mm}] \\
& \square \text { Maximum total } \\
& \text { deformation }[\mathrm{mm}]
\end{aligned}
$$

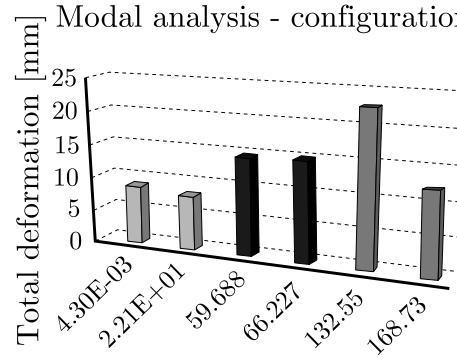

Frequency $[\mathrm{Hz}]$

\section{Minimum total deformation $[\mathrm{mm}]$ \\ $\square$ Maximum total deformation [mm]}

Fig. 14. Graphs of results of modal analysis of all configuration of RCM mechanisms

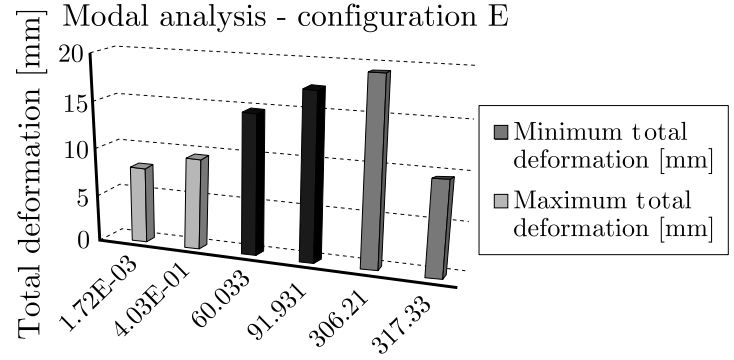

Frequency $[\mathrm{Hz}]$ 


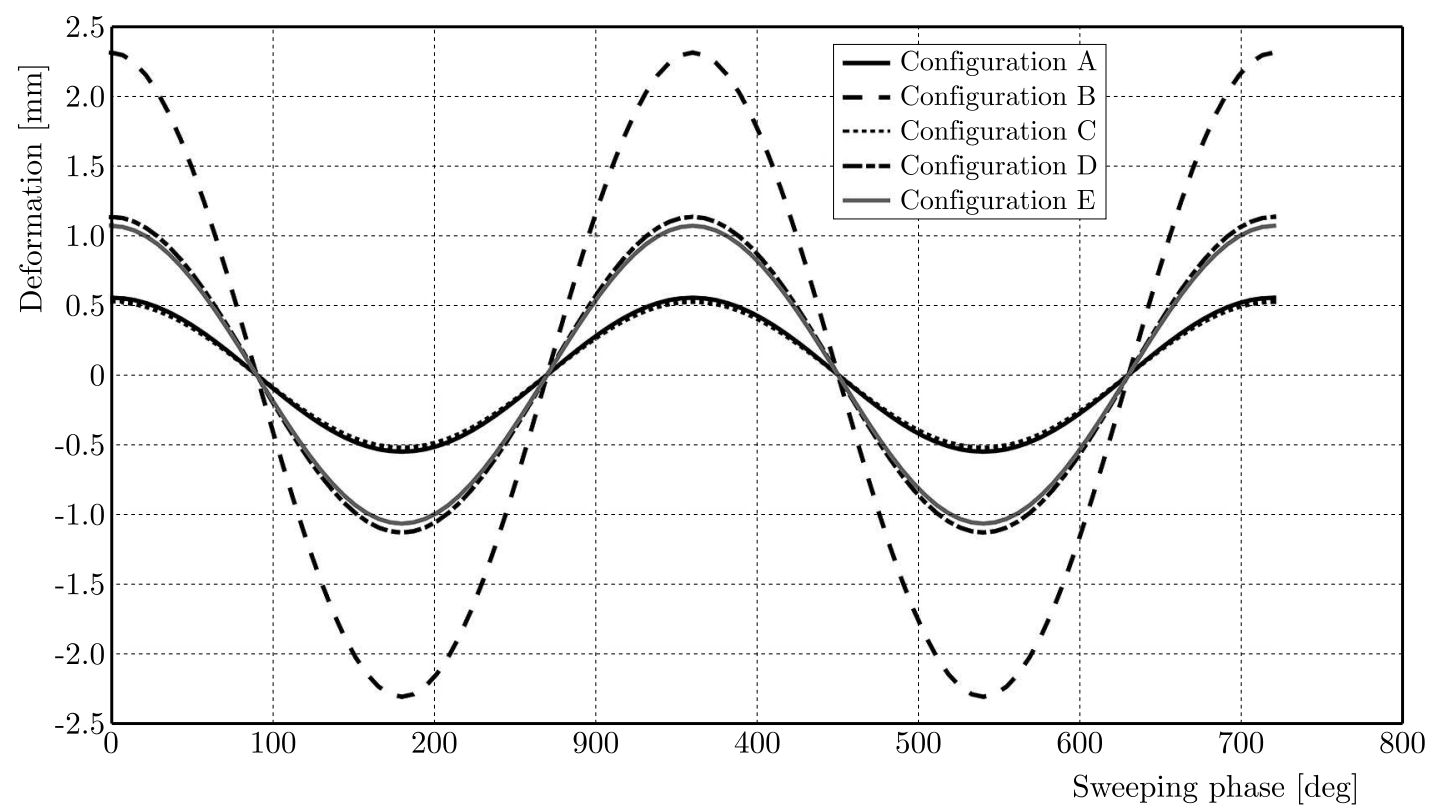

Fig. 15. Graphs of the comparative phase response of all RCM mechanisms

external forces, in order to quantify the magnitude of deformations in the structure of the mechanism, which has an influence on the change of TCP position.

- The most beneficial weight ratio of the tested mechanisms is obtained, in order from the best to worst, in configurations A, C, E, D, B; which simultaneously translates to lower values of potential, kinetic, total and internal energy compared to other configurations. Considering the best and worst case (configurations A and B), with regard to potential energy, the difference between the systems is $23.87 \%$ to the detriment of configuration B. Whereas the difference in kinetic energy is approx. 33.45\%, in total energy $24.09 \%$, in internal energy $24.74 \%$. Considering this criterion, a drive of lower power can be applied in configurations $\mathrm{A}, \mathrm{C}$ and $\mathrm{E}$ (the most favorable), which may affect the final price, weight and energy consumption required to control the mechanism. Configurations D and B are cast in an unfavorable light in this comparison. Considering this criterion, configuration A remains the best for selection as its total and internal energy is $2.33 \%$ lower than in configuration $\mathrm{C}$, and $3.92 \%$ lower than energy in configuration E.

- Modal analysis made it possible to determine the frequency of natural, unattenuated vibrations. Obtained results indicate that in the case of configurations A, B, C, the frequencies of natural vibrations with values of approx. $50-60 \mathrm{~Hz}$ may cause maximum deformations of the construction of the order of $10 \mathrm{~mm}$. For configurations D and E, at the same frequencies, deformations are nearly $47 \%$ larger. At a frequency of about $90 \mathrm{~Hz}$, deformation values of configurations $\mathrm{A}, \mathrm{C}, \mathrm{E}$ of the parallelogram reach up to approx. 16-18 mm. Configurations $\mathrm{B}$ and $\mathrm{D}$ have smaller deformations, however at a lower frequency, i.e. approx. $60 \mathrm{~Hz}$.

- Frequency responses of parallelogram mechanisms within the frequency range from 1 to $100 \mathrm{~Hz}$ (Figs. 12 and 13) indicate that it is most favorable to control the structures of the RCM mechanisms in configurations $\mathrm{E}, \mathrm{A}, \mathrm{C}$ within the range from 1 to $55 \mathrm{~Hz}$. Within the range from $55 \mathrm{~Hz}-100 \mathrm{~Hz}$, the places where resonances may occur are observed. In this respect, configurations $\mathrm{B}, \mathrm{D}$ and $\mathrm{E}$ are the least favorable (in these configurations, the amplitudes exceed $1 \mathrm{~mm}$ in the worst case). At low frequencies up to $10 \mathrm{~Hz}$, in the case of configuration $\mathrm{B}$, high amplitude values (up to $1 \mathrm{~mm}$ ) are also visible. At a frequency of approx. $20 \mathrm{~Hz}$, configuration D also displays a significant amplitude, indicating the potential 
for resonance. Analysis of the evolutions of the phase angle allows for the statement that each of the tested configurations is stable, since periodic transitions of the phase angle within the range from $-180^{\circ}$ to $180^{\circ}$ are observed. A change of the end effector load does not cause significant changes in the amplitude of vibrations.

- Harmonic analysis made it possible to determine the phase-frequency response. It also allowed for determination of the actual amplitude reached by displacement during long exposure to the sinusoidally variable load lasting $720^{\circ}$. In the analyzed cases, configurations $\mathrm{A}$ and $\mathrm{C}$ appear to be the most favorable (in these cases, deformations are of the order of approx. 5.5e-04 mm). Configurations D and E are comparable but worse than the aforementioned. The worst-case is configuration $\mathrm{B}$, where deformations amount to approx. 2.3e-03 $\mathrm{mm}$ and are nearly one order of magnitude greater than in cases $\mathrm{A}$ and $\mathrm{C}$ ).

- In order the improve the accuracy of movements of the parallelogram mechanism in surgical robots, one can use calibration techniques currently applied to industrial robots. A description of a multilevel calibration technique can be found in the work by Guo et al. (2015).

- Optimal designing of a surgical manipulator with a parallelogram mechanism can be achieved with the application of numerical methods. The structure-control design methodology described in the work (Valdez et al., 2016) can also be used for this purpose.

\section{Conclusions}

The parallelogram-based RCM mechanism is a simple and effective solution that makes it possible to achieve fixed-point movements of a surgical robot end effector due to its simple structure and an easy method of position control (with only two drives). The low weight of the mechanism structure, high rigidity and, hence, high accuracy of the end effector movements, are rational arguments in favor of the application of solutions of this type in new designs of active manipulators in new surgical robots. The results of kinematic, dynamic numerical analyses as well as modal analysis conducted in this work indicate that the mechanism with configuration $\mathrm{A}$ is the most favorable structure. Alternatively, configurations C and D should also be considered for applications in new designs.

\section{Acknowledegements}

This work was performed within the framework of statutory research of the Department of Automatic Control and Robotics at Bialystok University of Technology No. WZ/WE-IA/4/2020 and financed with funds from the Ministry of Science and Higher Education, Poland.

\section{References}

1. Ansys Inc., 2013, Ansys Mechanical APDL Contact Technology Guide, Release 15.0

2. Bai G., Li D., Wei S., Liao Q., 2014, Kinematics and synthesis of a type of mechanisms with multiple remote centers of motion, Proceedings of the Institution of Mechanical Engineers, Part C: Journal of Mechanical Engineering Science, 228, 18, 3430-3440

3. Chen Z., Li J., Zhang G., Wang S., 2015, A class of 1 DOF planar RCM mechanism based on motion-reproduce method, IEEE International Conference on Robotics and Biomimetics (ROBIO), 1433-1438

4. Chng C.B., Dunan B., Chui C.K., 2016, Modeling and simulation of a Remote Center of Motion mechanism, Proceedings of Region 10 Conference (TENCON), 2016 IEEE, 1755-1758 
5. Choi H., Kim H.J., Lim Y.A., Kwak H.S., Jang J.W., Won J., 2013, Conically shaped remote center-of-motion mechanism for single-incision surgery, IEEE/RSJ International Conference on Intelligent Robots and Systems, 3604-3609

6. Fu Y., Niu G., Pan B., Li K., Wang S., 2013, Design and optimization of remote center motion mechanism of minimally invasive surgical robotics, IEEE International Conference on Robotics and Biomimetics (ROBIO), 774-779

7. Gijbels A., Reynaerts D., Vander Poorten E.B., 2014, Design of 4-DOF parallelogram-based RCM mechanisms with a translational DOF implemented distal from the end-effector, Mechanisms and Machine Science, 22, 103-111, DOI: 10.1007/978-3-319-07058-2_12

8. Guo Y., Yin S., Ren Y., Zhu J., YAng S., Ye S., 2015, A multilevel calibration technique for an industrial robot with parallelogram mechanism, Precision Engineering, 40, 261-272

9. Hadavand M., Mirbagheri A., Behzadipour S., Farahmand F., 2014, A novel remote center of motion mechanism for the force-reflective master robot of haptic tele-surgery systems, The International Journal of Medical Robotics and Computer Assisted Surgery, 10, 2, 129-139

10. Koseki Y., Koyachi N., Arai T., Chinzei K., 2003, Remote actuation mechanism for MR-compatible manipulator using leverage and parallelogram-workspace analysis, workspace control, and stiffness evaluation, Proceedings - IEEE International Conference on Robotics and Automation, 1, 652-657

11. KuO C.H., DAI J.S., 2009, Robotics for minimally invasive surgery: a historical review from the perspective of kinematics, Proceedings of International Symposium on History of Machines and Mechanisms, Springer, Dordrecht, 337-354

12. Kuo C.H., DAi J.S., DAsGupta P., 2012, Kinematic design considerations for minimally invasive surgical robots: an overview, The International Journal of Medical Robotics and Computer Assisted Surgery, 8, 2, 127-145

13. Lee H., Cheon B., Hwang M., Kang D., Kwon D.S., 2018, A master manipulator with a remote-center-of-motion kinematic structure for a minimally invasive robotic surgical system, The International Journal of Medical Robotics and Computer Assisted Surgery, 14, 1, 12

14. Li G.K., Essomba T., Wu C.T., Lee S.T., Kuo C.H., 2017, Kinematic design and optimization of a novel dual-orthogonal remote center-of-motion mechanism for craniotomy, Proceedings of the Institution of Mechanical Engineers, Part C: Journal of Mechanical Engineering Science, 231, 6, 1129-1145

15. LiU X.J., WAng J., 2003, Some new parallel mechanisms containing the planar four-bar parallelogram, The International Journal of Robotics Research, 22, 9, 717-732

16. PAN B., FU Y., NiU G., XU D., 2014, Optimization and design of remote center motion mechanism of Minimally Invasive Surgical robotics, 11th International Conference on Ubiquitous Robots and Ambient Intelligence (URAI), 3-6

17. Roh S.G., Lee Y., Lee J., Ha T., Sang T., Moon K.W., Choi J.Y., 2015, Development of the SAIT single-port surgical access robot slave arm based on RCM Mechanism, Proceedings of Engineering in Medicine and Biology Society (EMBC), 37th Annual International Conference of the IEEE, 5285-5290

18. Taylor R.H., Menciassi A., Fichtinger G., Fiorini P., Dario P., 2016, Medical Robotics and Computer-Integrated Surgery, Springer Handbook of Robotics, Springer, Cham, 1657-1684

19. Trochimczuk R., 2013, Conception of arm of medical robot dedicated to application of minimally invasive surgery, Solid State Phenomena, 198, 3-8

20. Trochimczuk R., Łukaszewicz A., Mikołajczyk T., Aggogeri F., Borboni A., 2019, Finite element method stiffness analysis of a novel telemanipulator for minimally invasive surgery, Simulation, 95, 11, 1015-1025, DOI: 10.1177/0037549719835920

21. Valdez S.I., Chávez-Conde E., Hernandez E.E., Ceccarelli M., 2016, Structure-control design of a mechatronic system with parallelogram mechanism using an estimation of distribution algorithm, Mechanics Based Design of Structures and Machines, 44, 1-2, 58-71 
22. Wang W., Li J., Wang S., Su H., Jiang X., 2016, System design and animal experiment study of a novel minimally invasive surgical robot, The International Journal of Medical Robotics and Computer Assisted Surgery, 12, 1, 73-84

23. Yip H.M., Li P., Navarro-Alarcon D., Wang Z., Liu Y.H., 2014, A new circular-guided remote center of motion mechanism for assistive surgical robots, IEEE International Conference on Robotics and Biomimetics (ROBIO), 217-222

24. Zhang Z., Yu H., Du Z., 2015, Design and kinematic analysis of a parallel robot with Remote Center of Motion for Minimally Invasive Surgery, IEEE International Conference on Mechatronics and Automation (ICMA), 698-703

25. Zong G., Pei X., Yu J., Bi S., 2008, Classification and type synthesis of 1-DOF remote center of motion mechanisms, Mechanism and Machine Theory, 43, 12, 1585-1595

Manuscript received November 4, 2018; accepted for print February 24, 2020 